\title{
AMCoR
}

Asahikawa Medical College Repository http://amcor.asahikawa-med.ac.jp/

Cancer Letters (2004. May) 208(2):215-225.

VEGF and bFGF expression and microvessel density of maxillary sinus squamous cell carcinoma in relation to p53 status, spontaneous apoptosis and prognosis

Bandoh, Nobuyuki ; Hayashi, Tatsuya ; Takahara, Miki ; Kishibe, Kan ; Ogino, Takeshi ; Katayama, Akihiro ; Imada, Masanobu ; Nonaka, Satoshi ; Harabuchi, Yasuaki 


\section{VEGF and bFGF expression and microvessel density of maxillary sinus squamous cell carcinoma in relation to 533 status, spontaneous apoptosis and prognosis}

Nobuyuki Bandoh, Tatsuya Hayashi, Miki Takahara, Kan Kishibe, Takeshi Ogino, Akihiro Katayama, Masanobu Imada, Satoshi Nonaka, Yasuaki Harabuchi* Department of Otolaryngology-Head and Neck Surgery, Asahikawa Medical College, 2-1-1-1 Midorigaoka Higashi, Asahikawa, 078-8510, Japan

*Corresponding Author: Yasuaki Harabuchi

Department of Otolaryngology-Head and Neck Surgery, Asahikawa Medical College, 2-1-1-1 Midorigaoka Higashi, Asahikawa, 078-8510, Japan.

Phone: +81-166-68-2554; Fax: +81-166-68-2559; E-mail: hyasu@asahikawa-med.ac.jp

Summary: VEGF and bFGF expression and tumor microvessel density in tumor lesions were analyzed in 70 patients with maxillary sinus squamous cell carcinoma. The VEGF expression dependent of p53 overexpression and/or mutations was associated with angiogenesis, decreased spontaneous tumor apoptosis and metastases, while the bFGF expression was associated with resistance to radiochemotherapy, resulting in poor prognosis.

Keywords: vascular endothelial growth factor; basic fibroblast growth factor; microvessel density; maxillary sinus squamous cell carcinoma; p53; spontaneous apoptosis; prognosis 


\section{Abstract}

We have previously reported that p53 mutations, loss of bax expression or decreased spontaneous tumor apoptosis were associated with poorer prognoses in maxillary sinus squamous cell carcinoma (SCC)(Cancer 94: 1968-80, 2002). In the present study, we analyzed tumor angiogenesis monitored by expression of vascular endothelial growth factor (VEGF), basic fibroblast growth factor (bFGF) and tumor microvessel density, in correlation with p53 status, spontaneous apoptosis or disease prognosis in the same group of 70 maxillary sinus SCC patients. Tumor biopsy specimens obtained prior to initiation of treatment were examined for expression of VEGF and bFGF and tumor microvessel density using immunohistological methods. Average vessel density (AVD) (range: 3-75; median: 25) and maximum vessel density (MVD) (range: 4-125; median: 53) were assessed by the number of microvessels stained with anti-CD31 mAb in tumor lesions. VEGF was expressed in $35(50 \%)$ of 70 patients and bFGF was in $43(61 \%)$. Patients with VEGF expression showed significantly higher levels of MVD than those without VEGF expression (57 vs. 38; $\mathrm{p}=0.019$ ). The VEGF expression was observed more frequently in patients with p53 overexpression and/or mutation than in those with normal p53 status $(p=0.048)$. The MVD inversely correlated with the apoptotic index (AI) defined as the number of single stranded (ss)-DNA-positive cells per 1000 tumor cells $(r=-0.23 ; p=0.022)$. Patients with neck lymph node and/or distant metastases after surgery showed significantly higher levels of MVD than patients without any metastasis (64 vs. $42 ; p=0.048$ ). Low 
histological effectiveness of radiochemotherapy correlated with bFGF expression $(\mathrm{p}=0.0059)$. To clarify actual prognostic factors for maxillary sinus SCC, we selected 57 patients treated uniformly with preoperative radiochemotherapy followed by maxillectomy. Kaplan-Meier analysis showed that survival was significantly worse in patients with high $\operatorname{MVD}(\geq 80)$ than in those with low MVD $(<80)(\mathrm{p}=0.042)$. These data suggest that the VEGF expression in association with the p53 overexpression and/or mutations may cause increased microvascularity, decreased spontaneous apoptosis or metastases, while the bFGF expression may be associated with resistance to radiochemotherapy, thereby resulting in poorer prognoses in maxillary sinus SCC. 


\section{Introduction}

Angiogenesis, the formation of new capillaries out of pre-existing vessels, plays a crucial role in solid tumor growth $[1,2]$ and facilitates tumor progression and metastasis $[3,4]$. Angiogenesis is regulated by a large number of angiogenic factors, including vascular endothelial growth factor (VEGF) and basic fibroblast growth factor (bFGF). Although tumor-derived bFGF has both autocrine and paracrine functions, VEGF is produced only by tumors for the stimulation of tumor vascularization. VEGF is a disulfide-linked dimeric glycoprotein that increases blood vessel permeability and promotes endothelial cell growth, proliferation, migration and differentiation [5]. bFGF is involved in proliferation and differentiation of a wide range of cell types and exhibits a potent angiogenic activity [1].

In head and neck squamous cell carcinoma (SCC), a number of reports have been documented VEGF expression [6-13], bFGF expression [14-16] and tumor microvessel density [11,12,17-23]. Some reports showed that expression of VEGF [11,24] and bFGF [25] was associated with an increase in the tumor microvessel density. However, conflicting results have been presented with regard to the correlation of VEGF and bFGF expression with disease prognosis or tumor growth. Some studies have demonstrated that there is a significant correlation of VEGF and bFGF expression with poorer prognoses $[6,10,12-15]$ whereas others have shown no correlation $[7-9,11,16]$. On the other hand, many other studies have demonstrated a close association of tumor microvessel density with poorer prognoses $[12,17,18,21-23]$ although a few have shown no association [11,19]. In general, 
these studies of head and neck SCC lesions have lumped together tumors with different anatomic sites within the head and neck, with the exception of certain studies on laryngeal SCC patients $[9,11,22]$. Since variations in clinical and/or etiological behaviors may exist between different sites in the head and neck SCC, analysis from a single anatomic site is warranted. However, this has not been fully studied in maxillary sinus SCC as of yet.

Previously, we showed that p53 mutations, loss of bax expression, decreased spontaneous tumor apoptosis or low histological effectiveness of radiochemotherapy were significantly correlated with poorer prognoses in maxillary sinus SCC [26]. Furthermore, we found that bax expression was associated with increased spontaneous tumor apoptosis, while decreased spontaneous tumor apoptosis and p53 mutations correlated with low histological effectiveness of radiochemotherapy, suggesting that higher levels of spontaneous tumor apoptosis induced by bax expression may increase the sensitivity of tumors to radiochemotherapy, thereby resulting in good prognoses, while p53 mutations may lead to resistance against radiochemotherapy, resulting in poor prognoses. Recently, it has been reported that VEGF expression correlates with p53 overexpression and/or mutations in noninvasive colorectal carcinoma [27], lung carcinoma [28], primary breast carcinoma [29] and head and neck SCC [16]. It is also reported that VEGF expression and/or high tumor microvessel density correlate with lower levels of tumor apoptosis in gastric carcinomas [30-32], esophageal SCC [33] and head and neck SCC [34]. A recent in vitro study showed that bFGF expression was associated with resistance to 
chemotherapeutic agents in a human bladder cancer cell line [35].

The present study is the first attempt to examine the VEGF and bFGF expression and tumor microvessel density using immunohistological methods, and to investigate correlations of these angiogenic factors with p53 status, tumor apoptosis, the effectiveness of radiochemotherapy and disease prognosis in maxillary sinus SCC patients.

\section{Patients and methods}

\subsection{Patient characteristics}

The study group consisted of 70 Japanese patients (49 males and 21 females; age range: 36-86, median age: 67 ) who were treated for maxillary sinus SCC in the Department of Otolaryngology-Head and Neck Surgery, Asahikawa Medical College, between 1980 and 2000. All patients signed informed consents for therapy and tissue studies, which had received prior approval from the Institutional Review Board. This group had been previously studied for expression of apoptosis-related proteins including p53, Fas, bax and $\mathrm{bcl}-\mathrm{x}_{\mathrm{L}}$ as well as spontaneous tumor apoptosis and mutation in the p53 gene in relation to disease prognosis [26].

Clinical features, expression of apoptosis-related proteins and the p53 gene status

of the patients are listed in Table 1 [26]. According to the 1997 UICC TNM staging systems (identical to 1997 AJCC classification); T1 was not seen, T2 was present in 8 patients 
(11\%), T3 in 33 patients (47\%) and T4 in 29 patients (42\%). Seven patients $(10 \%)$ had lymph node metastasis (N1 in all cases) at diagnosis. Tumor differentiation was classified as well-differentiated in 29 patients (42\%), moderately in $24(34 \%)$ and poorly in $17(24 \%)$. Based on immunohistological analysis of biopsy tissues during the pretreatment period, p53 was overexpressed in $39(56 \%)$ of 70 patients, Fas was in $20(29 \%)$, bax was in $40(57 \%)$, and bcl- $\mathrm{x}_{\mathrm{L}}$ was in $33(47 \%)$. The apoptotic index (AI), defined as the number of single stranded (ss)-DNA-positive cells per 1000 tumor cells, ranged from 0.2 to 7.2 with median value of 1.8 [26]. Mutations of the p53 gene, which were analyzed by direct sequencing of PCR-amplified products obtained from laser microdissected tissues, were detected in 20 $(29 \%)$ patients.

Of 70 patients, 57 patients were treated with preoperative radiochemotherapy followed by total or partial maxillectomy. The preoperative radiochemotherapy including local irradiation with a total dose of 50 Gy (2.5 Gy x 20 fractions, 5 days a week) along with concomitant intra-maxillary arterial infusion of 5-fluorouracil (5FU) at a total dose of $5000 \mathrm{mg}$ (250 mg x 20 times) [36]. After the treatment, 9 patients developed neck lymph node and/or distant metastases. The remaining 13 patients were treated with radiotherapy alone because of inoperable intracranial invasion, advanced age with poor performance status, refusal of surgery, or comorbidities. Follow-up period of all patients ranged from 2 to 189 months with a median of 61 months. The 5-year overall and cause-specific survival rates for all patients were $48.5 \%$ and $75.7 \%$, respectively; for 13 patients who received 
radiotherapy alone, these rates were $13.1 \%$ and $0 \%$, respectively. For 57 patients who underwent preoperative radiochemotherapy followed by total or partial maxillectomy, the follow-up period ranged from 2 to 189 months with a median of 65 months ( 89 months for surviving patients). Of these 57 patients, 24 (42\%) patients had died during follow-up, including $8(14 \%)$ of tumor-related death and $16(28 \%)$ of death from comorbidities. The 5year overall and cause-specific survival rates were $55.6 \%$ and $81.2 \%$, respectively [26].

We have examined histological effectiveness of radiochemotherapy in surgically resected specimens obtained from 57 patients treated with radiochemotherapy followed by maxillectomy [26]. Histological effectiveness was classified into 5 grades according to the evaluation system proposed by Shimosato et al. [37] as follows: Grade 0: No characteristic changes in tumor cells and tumor structures. Grade I: Characteristic changes in tumor cells, but tumor structures have not been destroyed. Grade II: In addition to characteristic cellular changes, tumor structures have been destroyed as a result of the disappearance of tumor cells. However, variable numbers of viable tumor cells still remain. Grade III: Markedly altered and presumably nonviable tumor cells are present singly or in small clusters and viable tumor cells are hardly seen. Grade IV: No tumor cells remain in any section.

\subsection{Immunohistological analysis}

The formalin-fixed and paraffin-embedded tissues were obtained from transoral biopsy during the pretreatment period. The tissues were cut in $5 \mu \mathrm{m}$ sections. The sections 
were deparaffinized in xylene and dehydrated with ethanol. These sections were incubated with $3 \%$ hydrogen peroxidase in methanol for 30 minutes. Antigens were retrieved by microwave in $10 \mathrm{mM}$ citric acid buffer, $\mathrm{pH} 6.0$ for 5 minutes. The following antibodies were used: (a) anti-VEGF monoclonal antibody (mAb) (clone sc-7269, diluted 1:50; Santa Cruz Biotechnology, Inc., Santa Cruz, CA); (b) anti-bFGF polyclonal Ab (diluted 1:100; Santa Cruz Biotechnology, Inc.); (c) anti-CD31 mAb (clone JC/70A, diluted 1:20; Dako, Glostrup, Denmark) for the detection of microvessels. Tissue sections were sequentially incubated with mAbs overnight at $4^{\circ} \mathrm{C}$ and with peroxidase-labeled dextran polymer (EnVision ${ }^{+}$; Dako, Carpinteria, CA) for 30 minutes at room temperature. The sections were visualized by immersing the slides in freshly prepared $0.02 \%$ diaminobenzidine (DAB) solution for 10 minutes. The sections were finally counterstained with hematoxylin and mounted.

Stained tissue sections were examined microscopically in a coded manner by three of the authors (N.B., M.T., Y.H.), who had no knowledge of the patients and their clinical outcomes. More than 5000 tumor cells in at least 5 high power fields (x200) were counted to calculate the VEGF- and bFGF-positive cells and tumor microvessel density. VEGF and bFGF positivity were noted when the cytoplasm of tumor cells was strongly stained. The percentage of VEGF- and bFGF-positive tumor cells was expressed as a ratio of positive cells to the total number of tumor cells counted. In accordance these data together with previous reports $[16,25]$, tumors with $>25 \%$ of VEGF- and bFGF-positive cells were 
considered to be positive. To examine the tumor microvessel density, tissue sections were stained with anti-CD31 mAb. Average vessel density (AVD) was defined as the average of the number of CD31-positive microvessels in 5 fields $\left(x 200,0.94 \mathrm{~mm}^{2}\right)$. Then, slides were scanned at low power magnification (x100). In each tumor, "hot spot" areas displaying the highest vessel density were selected, as described previously [4]. The maximum vessel density (MVD) was determined from these "hot spot" areas at fields under high power magnification $\left(\mathrm{x} 200,0.94 \mathrm{~mm}^{2}\right)$. Sections stained with anti-mouse IgG1 mAb were used as negative controls.

\subsection{Statistical analysis}

Two group comparisons were tested using the Mann-Whitney U test and Fisher's exact test and summarized with their appropriate p-values. Cause-specific survival time was measured from the date of surgical removal of tumor to the date of death due to the original disease or final visit. The probability of the cause-specific survival was calculated using the Kaplan-Meier method and compared using the log-rank test.

\section{Results}

3.1. VEGF and bFGF expression and tumor microvessel density

VEGF was expressed in $35(50 \%)$ of 70 patients and bFGF was in $43(61 \%)$ (Fig. 
1). No significant correlation was found between VEGF and bFGF expression (Table 2). The AVD ranged from 3 to 75 with median value of 25 . The MVD ranged from 4 to 152 with median value of 53. No significant correlation was found between tumor microvessel densities, whether expressed as the AVD or MVD, and bFGF expression. However, patients with VEGF expression showed significantly higher levels of MVD than patients without VEGF expression (57 vs. 38; $\mathrm{p}=0.019$; Fig. 2), The expression of VEGF and bFGF as well as the tumor microvessel density did not correlate with either age, tumor differentiation or TNM classification prior to initiation of treatment.

3.2. VEGF and bFGF expression and tumor microvessel density in relation to p53 status As described in our previous report [26], 39 (56\%) of 70 patients showed p53 overexpression and $20(29 \%)$ had mutation of the p53 gene. Forty-three (61\%) showed abnormal p53 status, i.e., p53 overexpression and/or mutation. VEGF expression was observed significantly more frequently in patients with abnormal p53 status than in those with normal p53 status (61\% vs. 33\%; p=0.048; Table 2). The bFGF tended to be expressed more frequently in the patients with abnormal p53 status than in those with normal p53 status $(74 \%$ vs. $51 \% ; \mathrm{p}=0.082)$.

3.3. VEGF and bFGF expression and tumor microvessel density in relation to spontaneous tumor apoptosis 
None of the angiogenesis-associated variables correlated with the expression of Fas, bax, or bcl- $\mathrm{x}_{\mathrm{L}}$. However, VEGF expression tended to be associated with low AI $(<2)$ ( $p=0.054$; Table 2). The AVD as well as MVD were significantly higher in the patients with low $\mathrm{AI}(<2)$ than in those with high AI ( $\geq 2)$ (AVD: 41 vs. 29, p=0.017; MVD: 42 vs.28, $\mathrm{p}=0.009$; Fig. 3). The MVD inversely correlated with AI ( $\mathrm{r}=-0.23 ; \mathrm{p}=0.022 ;$ Fig. 4). The bFGF expression did not correlate with AI.

3.4. VEGF and bFGF expression and tumor microvessel density in relation to histological effectiveness of radiochemotherapy

Surgical specimens obtained from 57 patients with preoperative radiochemotherapy followed by total or partial maxillectomy were investigated for the histological effectiveness of radiochemotherapy. According to Shimosato's classification [37], Grade II was found in 21 patients (37\%), Grade III in 20 (35\%) and Grade IV in 16 (28\%). Neither Grade 0 nor Grade I was identified [26]. As shown in Table 3, only 5 (17\%) of 30 patients with bFGF expression showed a complete absence of tumor cells in the surgically resected tissues (Grade IV), while 11 (50\%) of 22 patients without bFGF expression $(\mathrm{p}=0.0059)$. No significant correlation was found between the histological effectiveness of radiochemotherapy and either VEGF expression or tumor microvessel density. 
3.5. VEGF and bFGF expression and tumor microvessel density in relation to metastases and prognosis

To clarify the actual prognostic values of VEGF and bFGF expression and tumor microvessel density for maxillary sinus SCC, we selected 57 patients treated uniformly with preoperative radiochemotherapy followed by total or partial maxillectomy and examined variables. After the treatment, 9 patients developed neck lymph node and/or distant metastases. The MVD in patients with neck lymph node and/or distant metastases was significantly higher than in those without any metastases (64 vs. 42; $p=0.048$; Fig. 5). Kaplan-Meier analysis showed that cause-specific survival was significantly worse in patients with high MVD $(\geq 80)$ than in those with low MVD $(<80)(p=0.042$; Fig. 6$)$. Kaplan-Meier analysis showed that cause-specific survival was significantly worse in patients with high MVD $(\geq 80)$ than in those with low MVD $(<80)(p=0.042$; Fig. 6). The VEGF and bFGF expression did not significantly correlate with either metastases or causespecific survival.

\section{Discussion}

Angiogenesis, which is regulated by a number of angiogenic factors, plays a crucial role in solid tumor growth $[1,2]$ and facilitates tumor progression and metastasis $[3,4]$. Among angiogenic factors, VEGF is generally regarded as the most potent factor and 
the most likely candidate for the induction of tumor growth, because it is a soluble secreted endothelial cell mitogen and its receptor such as kinase insert domain containing receptor (KDR) is selectively expressed by activated endothelium [38,39]. Angiogenesis can be monitored in a variety of ways including the tumor microvessel density. Some reports showed that VEGF expression correlated with increased tumor microvessel density in head and neck SCC [24] and laryngeal SCC [11]. Our study also showed that VEGF expression was closely associated with angiogenesis assisted by tumor microvessel density in maxillary sinus SCC.

It has been reported that VEGF expression correlated with p53 overexpression in noninvasive colorectal [27], lung [28] and primary breast carcinoma [29]. In head and neck SCC, Riedel et al. [16] demonstrated a significant correlation between p53 mutation and VEGF expression. Our present study clearly showed that abnormal p53 status, i.e., p53 overexpression and/or p53 gene mutation, was closely associated with VEGF expression in maxillary sinus SCC. These findings suggest that VEGF expression may be at least partly regulated by the p53 gene function. The hypothesis is supported by several lines of in vitro data [40-42]. Kieser et al. [40] demonstrated that mutant p53 could enhance VEGF expression induced by tumor promoting phorbol esters, such as 12-O-tetradecanoylphorbol-13-acetate, via activation of protein kinase C. Mukhopadhyay et al. [41] showed that wild-type p53 represses v-Src-mediated VEGF upregulation. Recently, Bouvet et al. [42] showed that transduction of p53-negative colon carcinoma cells with an 
adenoviral vector encoding wild-type p53 can decrease tumor growth by inhibiting VEGF mRNA expression.

It was reported in both in vivo and in vitro studies that angiogenic inhibition induces and sustains the dormancy of experimental tumors and micrometastases by increasing apoptotic cell death in tumor cells $[43,44]$. In surgically resected tumor tissues of gastric carcinomas [30-32] and esophageal SCC [33], VEGF expression and/or a high tumor microvessel density were reported to correlate with lower levels of apoptosis. In head and neck SCC, Riedel et al. [34] recently showed that high tumor vascularity correlated with low expression of pro-apoptotic factors such as FasL and caspase-3. In the present series of maxillary sinus SCC, we found that VEGF expression and higher tumor microvessel density were associated with lower levels of spontaneous tumor apoptosis. These data suggest that an inverse correlation between angiogenesis and apoptosis may exist in some malignancies including maxillary sinus SCC. This may be explainable by the close association between VEGF expression and abnormal p53 status, since VEGF induces angiogenesis and p53 mutation inhibits apoptosis. Hypoxia may be a link between apoptosis and angiogenesis. Hypoxia is a potent inducer of wild type p53 activity [45] and a strong relationship has been found between wild type p53 activity and stress-induced apoptosis [46]. Hypoxia may also induce anti-angiogenic regulators such as thrombospondin, presumably through the activity of p53 [47]. Alternatively, the inverse correlation between angiogenesis and apoptosis may be explainable by the anti-apoptotic 
function of VEGF by induction of bcl-2 expression, activation of the mitogen-activated protein kinase (MAPK) and inhibition of c-jun-N-terminal-kinase (JNK) [48,49].

Correlation between VEGF expression and bcl- $\mathrm{x}_{\mathrm{L}}$ expression observed here may support this possibility.

Although some conflicting results were reported [11,19], the majority of the reports in head and neck SCC showed that high tumor microvessel density correlated with tumor progression, recurrence, and/or poor prognosis [12,17,18,21-23]. The presence of high tumor microvessel density was reported to be useful as a predictor of metastases and/or poorer prognoses $[17,22,23]$. In our series of maxillary sinus SCC, high tumor microvessel density was significantly associated with neck lymph node and/or distant metastases as well as poorer prognoses.

There are some reports which indicate that bFGF expression correlated with tumor growth and metastasis in head and neck SCC $[14,15]$, but no correlation is also reported [16]. In the present series of maxillary sinus SCC, we found a significant correlation between bFGF expression and low effectiveness of radiochemotherapy. A recent in vitro study showed that bFGF expression as a result of transfection markedly suppresses the cisplatin-induced apoptosis in a human bladder cancer cell line, suggesting that bFGF expression may lead to resistance to chemotherapeutic agents [35]. The persistence of the disease after radiochemotherapy is usually a poor prognostic factor, since it is frequently associated with a higher risk of distant metastases and a worse prognosis [50]. Indeed, we 
have already found that the presence of tumor cells in surgically resected tissues after radiochemotherapy was closely related to poor disease-free survival in the same patients as those described in the present study [26]. Expression of bFGF is reported to be inhibited by p53 post-transcriptionally in human skin fibroblasts [51]. In this study, we showed that high bFGF expression tended to be associated with abnormal p53 status. It is indicated, on the basis of these data, that high bFGF expression, which is caused in part by abnormal p53 status, may be associated with poor prognosis via its resistant effect to radiochemotherapy in maxillary sinus SCC.

Some studies, in head and neck SCC other than maxillary sinus SCC, showed that VEGF expression correlated with tumor aggressiveness and/or poor prognosis $[6,10,12,13]$. In contrast, other studies showed no correlation of VEGF expression with tumor size, lymph node status, and/or prognosis $[7-9,11]$. These differences are likely to be caused by the different methods to assess VEGF expression and by lumping together tumors with different sites in head and neck lesion. In the present study of maxillary sinus SCC patients, VEGF expression did not directly correlate with the clinical outcome, but was significantly correlated with high tumor microvessel density, which is a significant indicator of poorer prognoses. Therefore, VEGF expression may be associated with poorer prognoses via the ability of VEGF to induce increased tumor microvascularity in maxillary sinus SCC lesions.

In combination with our previous study [26], we conclude that p53 mutations, 
decreased spontaneous tumor apoptosis and high tumor microvessel density may be useful prognostic factor for predicting long-term outcomes in maxillary sinus SCC patients. VEGF expression in association with abnormal p53 status may lead to increased tumor microvascularity and decreased tumor apoptosis, while bFGF expression may lead to resistance against radiochemotherapy. Because these molecular events contribute to poorer prognoses, they may also serve as candidate therapeutic targets in anti-cancer strategies.

\section{Acknowledgements}

We gratefully thank Mr. Eric Ko (Roswell Park Cancer Institute, Buffalo, NY) for excellent advice in the use of English. This work was supported by a Grant-in-Aid (No. 11470353) for scientific research from the Ministry of Education, Science and Culture, Japan.

\section{References:}

[1] J. Folkman and M. Klagsbrun, Angiogenic factors, Science 235 (1987) 442-447.

[2] J. Folkman, Angiogenesis in cancer, vascular, rheumatoid and other disease, Nat Med 1 (1995) 27-31.

[3] N. Weidner, J. P. Semple, W. R. Welch and J. Folkman, Tumor angiogenesis and metastasis--correlation in invasive breast carcinoma, N Engl J Med 324 (1991) 1-8.

[4] N. Weidner, J. Folkman, F. Pozza, P. Bevilacqua, E. N. Allred, D. H. Moore, S. Meli and G. Gasparini, Tumor angiogenesis: a new significant and independent prognostic 
indicator in early-stage breast carcinoma, J Natl Cancer Inst 84 (1992) 1875-1887.

[5] D. R. Senger, L. Van de Water, L. F. Brown, J. A. Nagy, K. T. Yeo, T. K. Yeo, B. Berse, R. W. Jackman, A. M. Dvorak and H. F. Dvorak, Vascular permeability factor (VPF, VEGF) in tumor biology, Cancer Metastasis Rev 12 (1993) 303-324.

[6] M. Moriyama, S. Kumagai, S. Kawashiri, K. Kojima, K. Kakihara and E. Yamamoto, Immunohistochemical study of tumour angiogenesis in oral squamous cell carcinoma, Oral Oncol 33 (1997) 369-374.

[7] P. Salven, P. Heikkila, A. Anttonen, M. Kajanti and H. Joensuu, Vascular endothelial growth factor in squamous cell head and neck carcinoma: expression and prognostic significance, Mod Pathol 10 (1997) 1128-1133.

[8] T. Maeda, S. Matsumura, H. Hiranuma, A. Jikko, S. Furukawa, T. Ishida and H. Fuchihata, Expression of vascular endothelial growth factor in human oral squamous cell carcinoma: its association with tumour progression and p53 gene status, J Clin Pathol 51 (1998) 771-775.

[9] M. Burian, C. Quint and C. Neuchrist, Angiogenic factors in laryngeal carcinomas: do they have prognostic relevance?, Acta Otolaryngol 119 (1999) 289-292.

[10] R. J. Eisma, J. D. Spiro and D. L. Kreutzer, Role of angiogenic factors: coexpression of interleukin-8 and vascular endothelial growth factor in patients with head and neck squamous carcinoma, Laryngoscope 109 (1999) 687-693.

[11] C. Neuchrist, C. Quint, A. Pammer and M. Burian, Vascular endothelial growth factor 
(VEGF) and microvessel density in squamous cell carcinomas of the larynx: an immunohistochemical study, Acta Otolaryngol 119 (1999) 732-738.

[12] E. R. Sauter, M. Nesbit, J. C. Watson, A. Klein-Szanto, S. Litwin and M. Herlyn, Vascular endothelial growth factor is a marker of tumor invasion and metastasis in squamous cell carcinomas of the head and neck, Clin Cancer Res 5 (1999) 775-782.

[13] H. Mineta, K. Miura, T. Ogino, S. Takebayashi, K. Misawa, Y. Ueda, I. Suzuki, M. Dictor, A. Borg and J. Wennerberg, Prognostic value of vascular endothelial growth factor (VEGF) in head and neck squamous cell carcinomas, Br J Cancer 83 (2000) $775-781$.

[14] C. J. Hughes, J. A. Reed, R. Cabal, A. G. Huvos, A. P. Albino and S. P. Schantz, Increased expression of basic fibroblast growth factor in squamous carcinogenesis of the head and neck is less prevalent following smoking cessation, Am J Surg 168 (1994) 381-385.

[15] F. R. Dellacono, J. Spiro, R. Eisma and D. Kreutzer, Expression of basic fibroblast growth factor and its receptors by head and neck squamous carcinoma tumor and vascular endothelial cells, Am J Surg 174 (1997) 540-544.

[16] F. Riedel, K. Gotte, J. Schwalb, C. Schafer and K. Hormann, Vascular endothelial growth factor expression correlates with p53 mutation and angiogenesis in squamous cell carcinoma of the head and neck, Acta Otolaryngol 120 (2000) 105-111.

[17] G. Gasparini, N. Weidner, S. Maluta, F. Pozza, P. Boracchi, M. Mezzetti, A. Testolin 
and P. Bevilacqua, Intratumoral microvessel density and p53 protein: correlation with metastasis in head-and-neck squamous-cell carcinoma, Int J Cancer 55 (1993) 739744.

[18] J. K. Williams, G. W. Carlson, C. Cohen, P. B. Derose, S. Hunter and M. J. Jurkiewicz, Tumor angiogenesis as a prognostic factor in oral cavity tumors, Am J Surg 168 (1994) 373-380.

[19] T. G. Dray, N. J. Hardin and R. A. Sofferman, Angiogenesis as a prognostic marker in early head and neck cancer, Ann Otol Rhinol Laryngol 104 (1995) 724-729.

[20] U. K. Zatterstrom, E. Brun, R. Willen, E. Kjellen and J. Wennerberg, Tumor angiogenesis and prognosis in squamous cell carcinoma of the head and neck, Head Neck 17 (1995) 312-318.

[21] J. D. Murray, G. W. Carlson, K. McLaughlin, M. Pennington, M. Lynn, P. B. DeRose, J. K. Williams and C. Cohen, Tumor angiogenesis as a prognostic factor in laryngeal cancer, Am J Surg 174 (1997) 523-526.

[22] T. Kamijo, T. Yokose, T. Hasebe, H. Yonou, S. Sasaki, R. Hayashi, S. Ebihara, H. Miyahara, H. Hosoi and A. Ochiai, Potential role of microvessel density in predicting radiosensitivity of $\mathrm{T} 1$ and $\mathrm{T} 2$ stage laryngeal squamous cell carcinoma treated with radiotherapy, Clin Cancer Res 6 (2000) 3159-3165.

[23] Y. Ito, T. Kamijo, T. Yokose, M. Kawashima, T. Ogino, H. Ikeda, R. Hayashi, S. Sasaki and A. Ochiai, Microvessel density predicts the radiosensitivity of metastatic 
head and neck squamous cell carcinoma in cervical lymph nodes, Int J Oncol 19 (2001) 1127-1132.

[24] N. Wakisaka, Q. H. Wen, T. Yoshizaki, T. Nishimura, M. Furukawa, E. Kawahara and I. Nakanishi, Association of vascular endothelial growth factor expression with angiogenesis and lymph node metastasis in nasopharyngeal carcinoma, Laryngoscope 109 (1999) 810-814.

[25] F. Riedel, K. Gotte, W. Bergler, W. Rojas and K. Hormann, Expression of basic fibroblast growth factor protein and its down-regulation by interferons in head and neck cancer, Head Neck 22 (2000) 183-189.

[26] N. Bandoh, T. Hayashi, K. Kishibe, M. Takahara, M. Imada, S. Nonaka and Y. Harabuchi, Prognostic value of p53 mutations, bax, and spontaneous apoptosis in maxillary sinus squamous cell carcinoma, Cancer 94 (2002) 1968-1980.

[27] Y. Kondo, S. Arii, M. Furutani, S. Isigami, A. Mori, H. Onodera, T. Chiba and M. Imamura, Implication of vascular endothelial growth factor and p53 status for angiogenesis in noninvasive colorectal carcinoma, Cancer 88 (2000) 1820-1827.

[28] G. Fontanini, S. Vignati, M. Lucchi, A. Mussi, A. Calcinai, L. Boldrini, S. Chine, V. Silvestri, C. A. Angeletti, F. Basolo and G. Bevilacqua, Neoangiogenesis and p53 protein in lung cancer: their prognostic role and their relation with vascular endothelial growth factor (VEGF) expression, Br J Cancer 75 (1997) 1295-1301.

[29] B. Linderholm, B. Lindh, B. Tavelin, K. Grankvist and R. Henriksson, p53 and 
vascular-endothelial-growth-factor (VEGF) expression predicts outcome in 833 patients with primary breast carcinoma, Int J Cancer 89 (2000) 51-62.

[30] C. Lu, J. W. Rak, H. Kobayashi and R. S. Kerbel, Increased resistance to oncostatin M-induced growth inhibition of human melanoma cell lines derived from advancedstage lesions, Cancer Res 53 (1993) 2708-2711.

[31] M. Ikeguchi, J. Cai, N. Yamane, M. Maeta and N. Kaibara, Clinical significance of spontaneous apoptosis in advanced gastric adenocarcinoma, Cancer 85 (1999) 23292335.

[32] M. Ikeguchi, J. Cai, K. Fukuda, S. Oka, K. Katano, S. Tsujitani, M. Maeta and N. Kaibara, Correlation between spontaneous apoptosis and the expression of angiogenic factors in advanced gastric adenocarcinoma, J Exp Clin Cancer Res 20 (2001) 257-263.

[33] N. Koide, A. Nishio, T. Kono, K. Yazawa, J. Igarashi, H. Watanabe, Y. Nimura, K. Hanazaki, W. Adachi and J. Amano, Histochemical study of vascular endothelial growth factor in squamous cell carcinoma of the esophagus, Hepatogastroenterology 46 (1999) 952-958.

[34] F. Riedel, K. Gotte, W. Bergler and K. Hormann, Inverse correlation of apoptotic and angiogenic markers in squamous cell carcinoma of the head and neck, Oncol Rep 8 (2001) 471-476.

[35] H. Miyake, I. Hara, K. Gohji, K. Yoshimura, S. Arakawa and S. Kamidono, 
Expression of basic fibroblast growth factor is associated with resistance to cisplatin in a human bladder cancer cell line, Cancer Lett 123 (1998) 121-126.

[36] T. Hayashi, S. Nonaka, N. Bandoh, Y. Kobayashi, M. Imada and Y. Harabuchi, Treatment of maxillary sinus carcinoma, Cancer 92 (2001) 1495-1503.

[37] Y. Shimosato, S. Oboshi and K. Baba, Histological evaluation of effects of radiotherapy and chemotherapy for carcinomas, Jap J Clin Oncol 1 (1971) 19-35.

[38] N. Ferrara, The role of vascular endothelial growth factor in pathological angiogenesis, Breast Cancer Res Treat 36 (1995) 127-137.

[39] B. I. Terman and M. Dougher-Vermazen, Biological properties of VEGF/VPF receptors, Cancer Metastasis Rev 15 (1996) 159-163.

[40] A. Kieser, H. A. Weich, G. Brandner, D. Marme and W. Kolch, Mutant p53 potentiates protein kinase $\mathrm{C}$ induction of vascular endothelial growth factor expression, Oncogene 9 (1994) 963-969.

[41] D. Mukhopadhyay, L. Tsiokas and V. P. Sukhatme, Wild-type p53 and v-Src exert opposing influences on human vascular endothelial growth factor gene expression, Cancer Res 55 (1995) 6161-6165.

[42] M. Bouvet, L. M. Ellis, M. Nishizaki, T. Fujiwara, W. Liu, C. D. Bucana, B. Fang, J. J. Lee and J. A. Roth, Adenovirus-mediated wild-type p53 gene transfer downregulates vascular endothelial growth factor expression and inhibits angiogenesis in human colon cancer, Cancer Res 58 (1998) 2288-2292. 
[43] L. Holmgren, M. S. O'Reilly and J. Folkman, Dormancy of micrometastases: balanced proliferation and apoptosis in the presence of angiogenesis suppression, Nat Med 1 (1995) 149-153.

[44] M. S. O'Reilly, L. Holmgren, C. Chen and J. Folkman, Angiostatin induces and sustains dormancy of human primary tumors in mice, Nat Med 2 (1996) 689-692.

[45] T. G. Graeber, J. F. Peterson, M. Tsai, K. Monica, A. J. Fornace, Jr. and A. J. Giaccia, Hypoxia induces accumulation of p53 protein, but activation of a G1-phase checkpoint by low-oxygen conditions is independent of p53 status, Mol Cell Biol 14 (1994) 6264-6277.

[46] S. W. Lowe, H. E. Ruley, T. Jacks and D. E. Housman, p53-dependent apoptosis modulates the cytotoxicity of anticancer agents, Cell 74 (1993) 957-967.

[47] A. J. Giaccia, Hypoxic Stress Proteins: Survival of the Fittest, Semin Radiat Oncol 6 (1996) 46-58.

[48] K. Gupta, S. Kshirsagar, W. Li, L. Gui, S. Ramakrishnan, P. Gupta, P. Y. Law and R. P. Hebbel, VEGF prevents apoptosis of human microvascular endothelial cells via opposing effects on MAPK/ERK and SAPK/JNK signaling, Exp Cell Res 247 (1999) 495-504.

[49] J. E. Nor, J. Christensen, D. J. Mooney and P. J. Polverini, Vascular endothelial growth factor (VEGF)-mediated angiogenesis is associated with enhanced endothelial cell survival and induction of Bcl-2 expression, Am J Pathol 154 (1999) 375-384. 
[50] H. Suit, A. Allam, J. Allalunis-Turner, W. Brock, T. Girinsky, S. Hill, N. Hunter, L. Milas, R. Pearcey, L. Peters and et al., Is tumor cell radiation resistance correlated with metastatic ability?, Cancer Res 54 (1994) 1736-1741.

[51] B. Galy, L. Creancier, C. Zanibellato, A. C. Prats and H. Prats, Tumour suppressor p53 inhibits human fibroblast growth factor 2 expression by a post-transcriptional mechanism, Oncogene 20 (2001) 1669-1677. 


\section{Figure legends}

Fig. 1. Representative immunohistological staining of VEGF (a) and bFGF (b) expression and microvessels in a tumor lesion stained with anti-CD31 mAb (c) in maxillary sinus SCC. $x 200$.

Fig. 2. Average microvessel density (AVD) (O) and maximum vessel density (MVD) (•) in tumor lesions in patients with or without VEGF expression. The MVD in patients with VEGF expression was significantly higher than that in patients without VEGF expression (57 vs. 38; $\mathrm{p}=0.019)$. The median values are expressed as short bar (-). The Mann-Whitney U test was used to determine the p-value.

Fig. 3. Average microvessel density (AVD) (O) and maximum vessel density (MVD) (•) in tumor lesions in patients with low apoptotic index $(\mathrm{AI}<2)$ or with high $\mathrm{AI}(\geq 2)$. The MVD and AVD were significantly higher in the patients with low AI $(<2)$ than in the patients with high AI ( $\geq 2$ ) (MVD: 42 vs. 28, p=0.009; AVD: 41 vs.29, $p=0.017$, respectively). The median values are expressed as short bar (-). The Mann-Whitney U test was used to determine the corresponding p-values.

Fig. 4. Correlation between maximum vessel density (MVD) and apoptotic index (AI). The MVD inversely correlated with AI $(r=-0.23 ; \mathrm{p}=0.022)$. The spearman regression coefficient was used to determine the r-and p-value.

Fig. 5. MVD in patients with or without neck lymph node and/or distant metastases. MVD in patients with neck lymph node and/or distant metastases was significantly higher than 
that in patients without metastases ( 64 vs. $42 ; p=0.048)$. The median values are expressed as short bar (-). The Mann-Whitney U test was used to determine the p-value.

Fig. 6. Cause-specific survival according to MVD in 70 patients with maxillary sinus SCC. The survival was significantly worse in patients with high MVD $(\geq 80)$ than in patients with low MVD $(<80)(\mathrm{p}=0.042)$. The Kaplan-Meier method and log-rank test were used for this analysis. 
Table 1

Clinical features, p53 gene status and expression of apoptosis-related proteins in 70 patients with maxillary sinus squamous cell carcinoma

\begin{tabular}{|c|c|c|}
\hline \multicolumn{2}{|c|}{ Variables } & No. of patients (\%) \\
\hline \multirow[t]{2}{*}{ Gender } & Male & $49(70 \%)$ \\
\hline & Female & $21(30 \%)$ \\
\hline \multirow[t]{2}{*}{ Age (years) } & $<65$ & $29(41 \%)$ \\
\hline & $\geq 65$ & $41(59 \%)$ \\
\hline \multirow[t]{3}{*}{ Tumor extension } & $\mathrm{T} 2$ & $8(11 \%)$ \\
\hline & Т3 & $33(47 \%)$ \\
\hline & $\mathrm{T} 4$ & $29(42 \%)$ \\
\hline \multirow[t]{2}{*}{ Lymph node } & No & $63(90 \%)$ \\
\hline & N1 & $7(10 \%)$ \\
\hline \multirow[t]{4}{*}{ Clinical stage } & II & $8(11 \%)$ \\
\hline & III & $33(47 \%)$ \\
\hline & IVA & $28(41 \%)$ \\
\hline & IVC & $1(1 \%)$ \\
\hline \multicolumn{2}{|c|}{ Tumor differentiat well } & $29(42 \%)$ \\
\hline & moderately & $24(34 \%)$ \\
\hline & poorly & $17(24 \%)$ \\
\hline \multirow[t]{2}{*}{ p53 gene } & wild type & $50(71 \%)$ \\
\hline & mutated & $20(29 \%)$ \\
\hline \multirow[t]{2}{*}{ p53 protein } & negative & $31(44 \%)$ \\
\hline & positive & $39(56 \%)$ \\
\hline \multirow[t]{2}{*}{ Fas } & negative & $50(71 \%)$ \\
\hline & positive & $20(29 \%)$ \\
\hline \multirow[t]{2}{*}{ bax } & negative & $30(43 \%)$ \\
\hline & positive & $40(57 \%)$ \\
\hline \multirow[t]{2}{*}{ bcl- $x_{\mathrm{L}}$} & negative & 36 (53\%) \\
\hline & positive & 34 (47\%) \\
\hline \multirow[t]{2}{*}{ Apoptotic index } & $<2$ & 37 (53\%) \\
\hline & $\geq 2$ & $33(47 \%)$ \\
\hline
\end{tabular}


Table 2

Correlation among VEGF, bFGF and bcl- $\mathrm{x}_{\mathrm{L}}$ expression, p53 status and Apoptotic index in primary tumor samples obtained from 70 patients with maxillary sinus squamous cell carcinoma

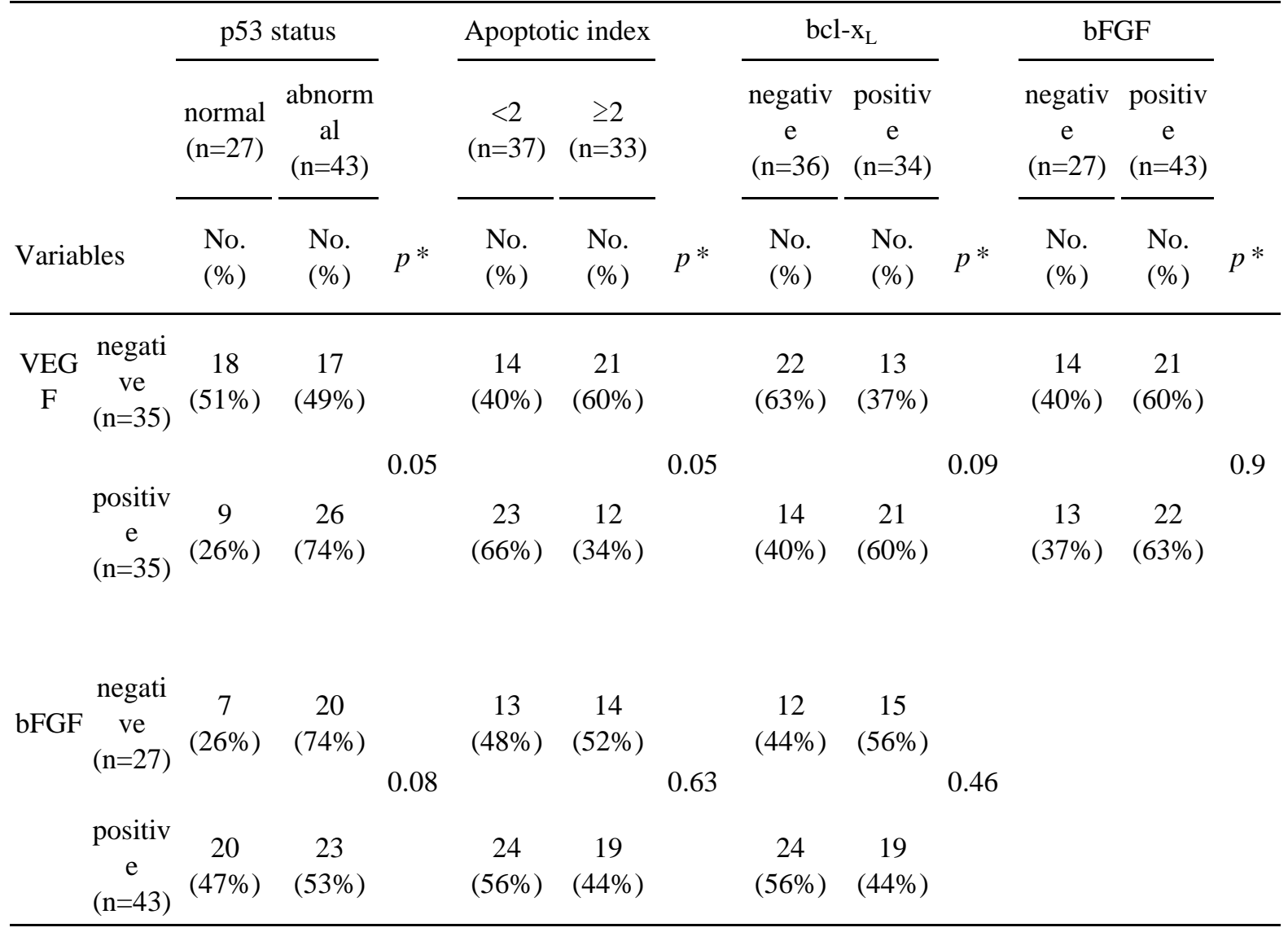

*p-values were determined by the Fisher's exact test. Abnormal p53 status means p53 overexpression and/or mutation. p53 gene status and expression of apoptosis-related proteins have been analyzed elsewhere [26]. 
Table 3

Correlation between bFGF expression and

histological effectiveness of radiochemotherapy

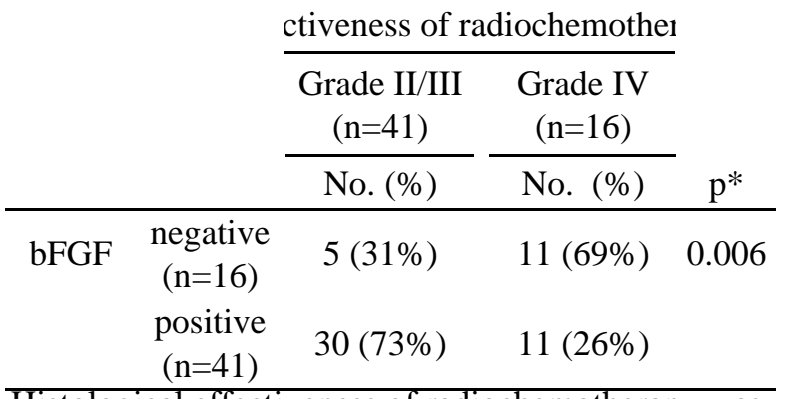

Histological effectiveness of radiochemotherapy was classfied as follows[37]: Grade II: In addition to

characteristic cellular changes, tumor structures have

been destroyed as a result of the disappearance of

tumor cells. However, variable number of viable

tumor cells still remain. Grade III: Markedly altered

and presumably nonviable tumor cells are present 

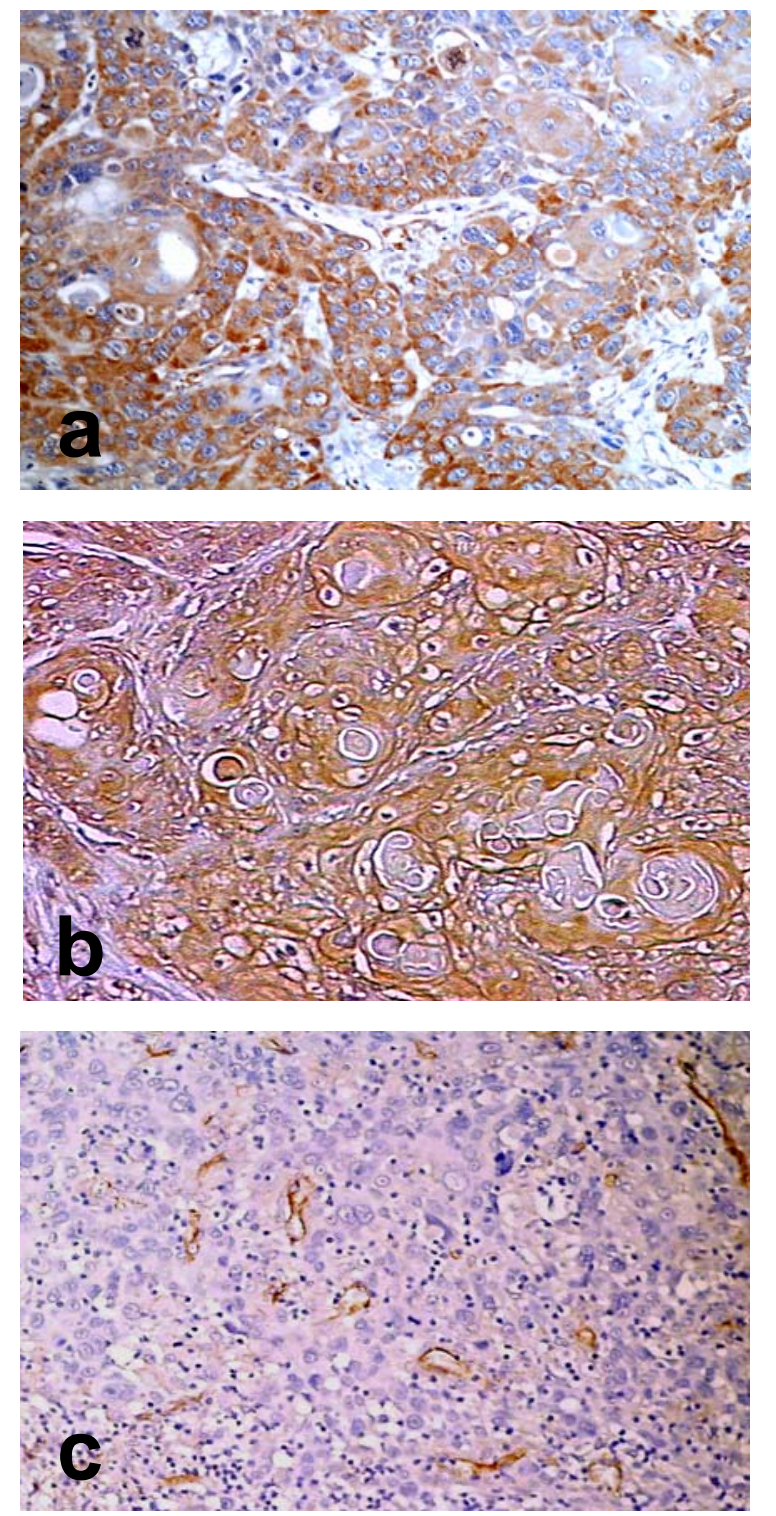
Fig. 2

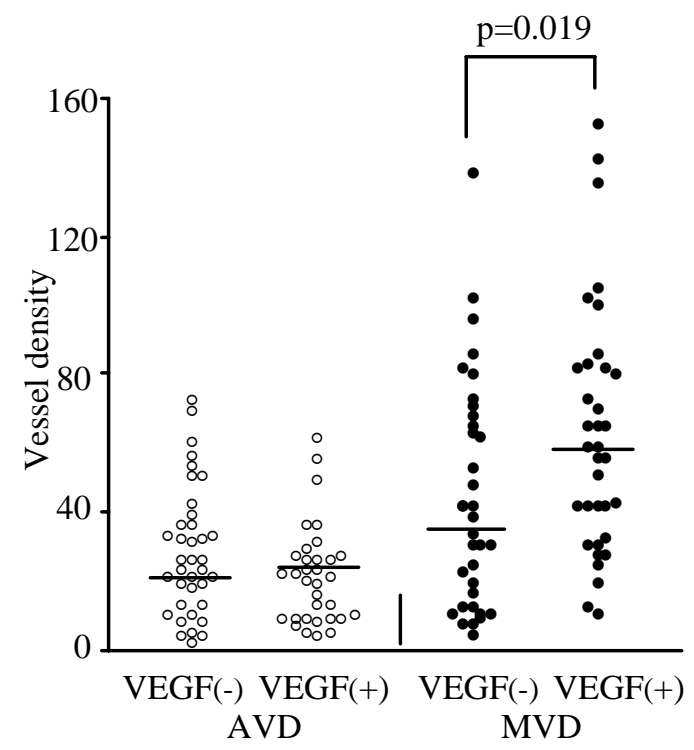


Fig. 3

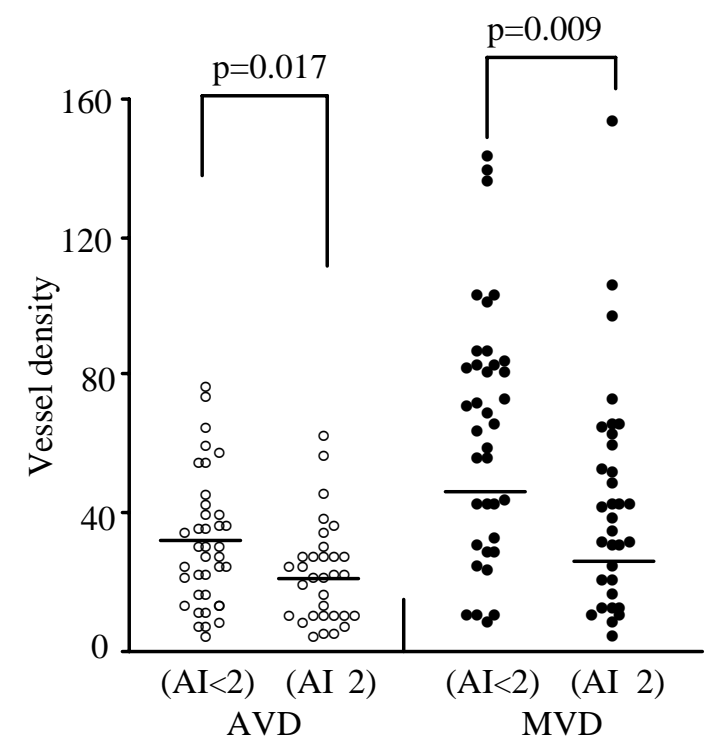


Fig. 4

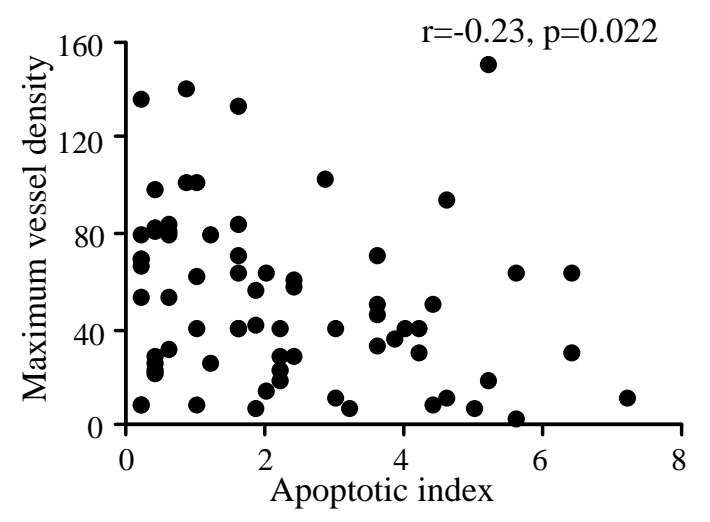


Fig. 5

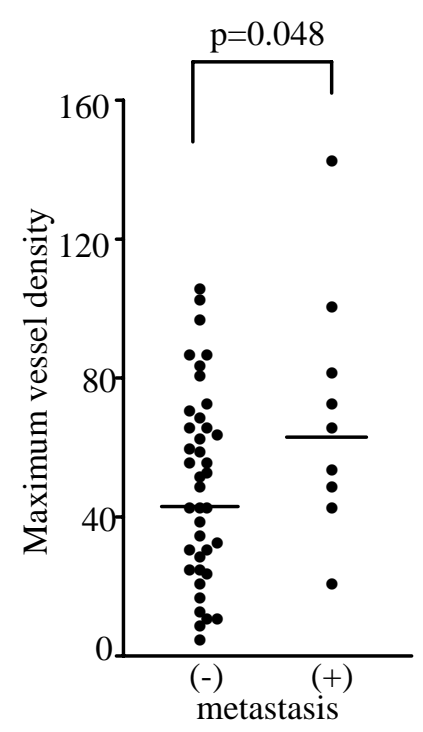


Fig. 6

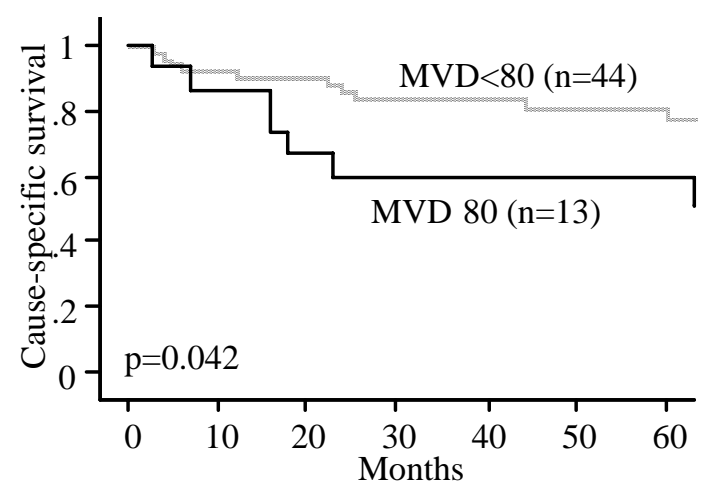

